\author{
MITSUBISHI ELECTRIC RESEARCH LABORATORIES \\ http://www.merl.com
}

\title{
Computing During Supply Voltage Switching in DVS Enabled Real-time Processors
}

\author{
Chunjie Duan, Sunil Khatri
}

TR2006-033 May 2006

\begin{abstract}
In recent times, much attention has been devoted to power optimization for real-time systems, while guaranteeing that such systems meet their hard (or soft) scheduling deadlines. To reduce power, different tasks in such systems may be run at different power supply voltages, in order to maximally utilize slack in the schedule. However, prior approaches have ignored the practical aspects of switching the power supply. In a typical IC, the VDD net is highly capacitive, and as a result, its vltage cannot be changed instantaneously. In traditional approaches, the assumption is that this net switches instaneously, which in effect makes it essential to include the VDD switching time in the worst-case execution time (WCET) or a process (adding pessimism to the WCET value). In our approach, we precisely model the switching of the VDD net, and allow the system to continue computations while VDD is being switched. the effect on the delay of tasks during this transition is precisely modeled. This allows a designer to obtain more realistic estimates of the WCET of a process, reducing the psssimism inherent in real-time system scheduling. Our approach can be implemented as a simple look-up table in a real-time scheduler. Our experimental results show that our model is highly accurate, with an error of less-than $0.2 \%$ compared to SPICE simulations.
\end{abstract}

IEEE International Symposium on Circuits and Systems

This work may not be copied or reproduced in whole or in part for any commercial purpose. Permission to copy in whole or in part without payment of fee is granted for nonprofit educational and research purposes provided that all such whole or partial copies include the following: a notice that such copying is by permission of Mitsubishi Electric Research Laboratories, Inc.; an acknowledgment of the authors and individual contributions to the work; and all applicable portions of the copyright notice. Copying, reproduction, or republishing for any other purpose shall require a license with payment of fee to Mitsubishi Electric Research Laboratories, Inc. All rights reserved. 



\title{
Computing During Supply Voltage Switching in DVS Enabled Real-time Processors
}

\author{
Chunjie Duan $^{\dagger} \quad$ Sunil P Khatri* \\ Mitsubishi Electric Research Laboratories, Cambridge, MA 02139 \\ * Department of EE, Texas A\&M University, College Station TX 77843.
}

\begin{abstract}
In recent times, much attention has been devoted to power optimization for real-time systems, while guaranteeing that such systems meet their hard (or soft) scheduling deadlines. To reduce power, different tasks in such systems may be run at different power supply voltages, in order to maximally utilize slack in the schedule. However, prior approaches have ignored the practical aspects of switching the power supply. In a typical IC, the VDD net is highly capacitive, and as a result, its voltage cannot be changed instantaneously. In traditional approaches, the assumption is that this net switches instantaneously, which in effect makes it essential to include the VDD switching time in the worst-case execution time (WCET) of a process (adding pessimism to the WCET value). In our approach, we precisely model the switching of the VDD net, and allow the system to continue computations while VDD is being switched. The effect on the delay of tasks during this transition is precisely modeled. This allows a designer to obtain more realistic estimates of the WCET of a process, reducing the pessimism inherent in real-time system scheduling. Our approach can be implemented as a simple look-up table in a real-time scheduler. Our experimental results show that our model is highly accurate, with an error of $<0.2 \%$ compared to SPICE simulations.
\end{abstract}

\section{Introduction}

The area of embedded system scheduling arguably started with the seminal work of Liu and Layland [1] in 1973. In this paper, the authors assumed a single-processor system with $n$ independent periodic tasks, and given worst case execution times (WCETs). Liu and Layland showed that if tasks were scheduled statically using a priority which was inversely proportional to their periodicity, the resulting schedule was optimum among all fixed priority schedules.

In recent times, the work of Liu and Layland has been extended in several ways. There have been several approaches to devise static power-conscious scheduling algorithms $[2,3,4,5]$. Other dynamic schedulers were also reported $[6,7,8,9,10]$ which utilized dynamic voltage scheduling (DVS). In a DVS processor, voltage may be modified dynamically, allowing the scheduler to trade off power for delay (by varying the VDD value of the processor). The independent task assumption of [1] was removed in [11]. Techniques to generate variable supply voltages were reported in $[12,13]$.

In the above scheduling algorithms, it is assumed that the delay overhead of VDD switching was negligible. However, this is not the case for realistic processors that are used to implement real time embedded systems. These processors have significantly capacitive VDD nets. For example, the capacitance on the VDD net in [14] was reported to be $160 \mathrm{nF}$. In fact, designers make special efforts to increase this capacitance for signal integrity reasons. This clearly makes the zero VDD switching delay assumption weaker. As a result, the assumption that VDD switching has negligible delay overhead is unjustified in modern designs. If we were to use the zero VDD switching delay assumption, the worst-case VDD switching delay must be included in the WCETs of each task, resulting in more conservative WCETs. If the computation of a task is pre-empted $n$ times by other tasks (operating at different voltages), we need to increment the WCET of the task by $n$ times the worst case VDD switching delay.

For these reasons, it would be desirable to have a methodology which reduces the conservatism of WCETs by modeling the VDD switching delay and accounting for it in the schedule. In this work, we present such a tech-

0-7803-9390-2/06/\$20.00 @2006 IEEE nique. Suppose a task $\varphi_{i}$ with scheduled voltage $V D D_{i}$ was completed and the next task $\varphi_{j}$ with scheduled voltage $V D D_{j}$ is started. Let $V D D_{i}<V D D_{j}$. Assume that task $\varphi_{j}$ had already been queued while $\varphi_{i}$ was being processed. In this case, we begin the VDD switching from $V D D_{i}$ to $V D D_{j}$ during the time $\varphi_{i}$ is being executed, which results in a condition where $\varphi_{i}$ completes its work (defined henceforth as the number of cycles required in the computation of a task) earlier. Task $\varphi_{j}$ now begins earlier than planned. We find the time $T_{1}^{*}$ at which, if VDD switching from $V D D_{i}$ to $V D D_{j}$ is initiated, then the speed-up of $\varphi_{i}$ is equal to the increased delay of $\varphi_{j}$. In other words, the work of both $\varphi_{i}$ and $\varphi_{j}$ completes before their respective deadlines. We formulate this problem and find an expression for the time $T_{1}^{*}$. We report the results of experiments to validate this expression, showing a close match between the mathematical model and the experimental delays.

Note that in the case that $V D D_{i}>V D D_{j}$, then the switching must be performed at the originally scheduled time (so that the work of $\varphi_{i}$ can be guaranteed to complete). Since the average value of VDD is higher during the computation of $\varphi_{j}$, it completes earlier than scheduled. Once again, we ensure that the required work for $\varphi_{i}$ and $\varphi_{j}$ is completed before their respective deadlines.

The rest of this paper is organized as follows. In Section 2, we discuss some prior work in real-time scheduling. Section 3 describes our approach, while Section 4 reports the results of experiments we conducted to validate our approach. Section 5 summarizes our work.

\section{Previous Work}

In the seminal paper by Liu and Layland [1], the authors motivated the area of real-time systems, and provided a fixed priority scheduler which had an asymptotic upper bound for processor utilization of $69 \%$. The focus of this work was schedulability, rather than power.

In recent times, with the growing interest in low-power real-time embedded systems, there have been several efforts to augment the work of [1] for low power applications. Most of these efforts attempt to reduce power by scaling the frequency of operation, the value of $\mathrm{VDD}^{1}$, or by powering down the system during periods of inactivity. An excellent review of low power scheduling is found in [15].

In [2], the authors augment a fixed priority schedule in a power conscious manner. If there is dead-time in the schedule, such periods are filled in by reducing the clock frequency, VDD value or by system power-down. In [3], the authors devised an algorithm to find the optimal voltage for each task. They ignore the delay and power overhead of switching VDD. However, this is a problem in general since the VDD net on an IC can be significantly capacitive, especially for Systems-on-a-Chip (SOCs). For an large IC, this capacitance can be in the range of a a few $100 \mathrm{nF}$ [14]. Later, in [4] an energy efficient fixed priority scheduling algorithm was reported, which could be used to find optimal voltages for each task or for entire task sets. Finally, in [5], a genetic DVS algorithm was presented.

Fixed priority dynamic voltage schedulers (DVS) have also been extended to dynamic schedulers. In [6], a DVS algorithm was reported for dynamic schedules, using slack analysis. In [7], the authors reported a static and dynamic algorithm for voltage and clock scaling of real-time embedded systems. In $[8,9]$, static as well as dynamic variable voltage schedulers were

${ }^{1}$ These techniques are classified as Dynamic Voltage Scaling (DVS) approaches. In these techniques, the VDD of the processor is reduced when there is slack in the schedule, thereby reducing the power. The VDD of a processor can be increased as well, resulting in a higher execution speed and 5115 ower consumption. 
reported for heterogeneous real-time distributed embedded systems.

The independent task assumption was removed in [11], where the authors reported a scheduling algorithm for periodic task graphs, with the additional ability to handle aperiodic tasks. These algorithms were generalized to the DVS scenario as well.

In [16], a battery aware static scheduler was presented, including an algorithm for voltage scalable processing elements (PEs). It was assumed that the PEs can perform voltage scaling. Such a capability is available in the Intel XScale processor [17] (in which voltage is continuously scalable) and the Transmeta Crusoe [18] (in which voltage is scalable in discrete steps).

In all the above efforts, the thrust was on scheduling algorithms. The time required to switch VDD was ignored, and implicitly included in the task WCET. This adds pessimism to the schedule, since the worst-case time taken by the VDD net to switch must be factored into the WCET of each process. In our work, we allow task execution during VDD switching, allowing the WCET value to be more realistic for real applications. This is a circuit oriented approach to DVS based scheduling, in which we are able to eliminate the overheads of VDD switching. Since our approach is independent of the scheduling algorithms used, it can be used along with any of the above scheduling algorithms. The contribution of this paper is to describe a technique where the traditional pessimism in WCET is reduced. The results from this paper can be applied to any of the scheduling algorithms in practice today.

Techniques to generate variable supply voltages were described in [12, 13]. These works describe solid state DC-DC regulators which could be used in VDD-scaled real-time systems. Our approach would work with either kind of regulator. Of course, the voltage regulator that is used can be offchip as well.

\section{Our Approach}

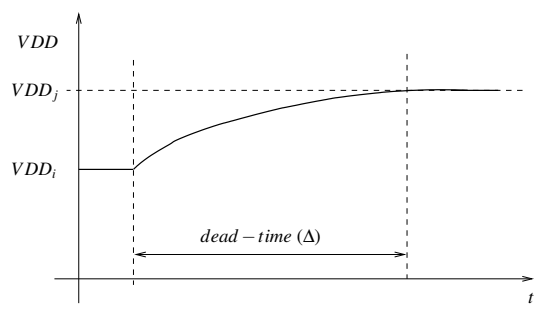

Figure 1: DVS timing diagrams

Figure 1 illustrates the problem being addressed by our approach. When a DVS enabled processor switches from $V D D_{i}$ to $V D D_{j}$ during operation, its switching waveform is a rising (or falling) exponential, since the VDD net on a modern IC is significantly capacitive, and the voltage regulation circuit has a finite series resistance. In this section, we discuss both the cases, in which $V D D_{i}<V D D_{j}$ and $V D D_{i}>V D D_{j}$. Traditional scheduling approaches consider the VDD transition to be an ideal step function, which means that the worst case dead-time $(\Delta)$ during VDD switching increases the WCET of each process. With typical values (VDD net capacitance $\sim$ $100 \mathrm{nF}$, supply resistance $\sim 1 \Omega$ ), we find that this dead-time can be in the $100 \mathrm{~ns}$ range. This adds to the pessimism in the WCET value. Since the capacitance of the VDD net is quite high, the pessimism introduced can be quite high, especially when a process is repeatedly interrupted by other processes (which compute at different VDD values). If in fact the process is interrupted $n$ times by other processes running at different VDDs, then its WCET must be increased by $n$ times the worst case VDD switching delay.

Consider the VDD switching waveforms in Figure 2. This figure shows two tasks $\varphi_{i}$ and $\varphi_{j}$ that are scheduled contiguously. $\varphi_{i}$ starts at $t=0$ and both tasks must be completed before $t=T_{2}$. The WCETs of $\varphi_{i}$ and $\varphi_{j}$ are $C_{i}$ and $C_{j}$ respectively and their supply voltages are $V D D_{i}$ and $V D D_{j}$ respectively. Assume that $V D D_{i}<V D D_{j}$. The deadline for $\varphi_{i}$ is $T_{1}$, and that of $\varphi_{2}$ is $T_{2}$. Assume that $\varphi_{j}$ is queued already.

Subfigure a) illustrates the ideal case (i.e. the VDD switching waveform is an ideal step function). In other words, this assumes that the dead-time $(\Delta)$ is zero.

Subfigure b) illustrates the actual VDD switching waveform, which our method incorporates into the schedule. Our method must obviously meet 116

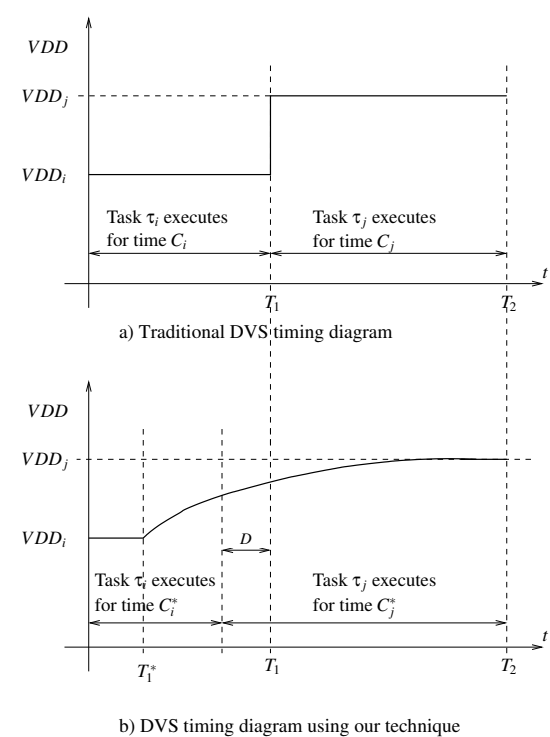

Figure 2: DVS timing diagrams

the deadlines $T_{1}$ and $T_{2}$. As we can see in the figure, because the rising time is significant, $\varphi_{j}$ will not complete in time if the switching from $V D D_{i}$ to $V D D_{j}$ starts at the same time as was used in the ideal case of Figure 2a). In our approach, we start the VDD transition from $V D D_{i}$ to $V D D_{j}$ at time $T_{1}^{*}$, (during the execution of task $\varphi_{i}$ ). As a consequence, the average $V D D$ value for task $\varphi_{i}$ increases above $V D D_{i}$, so that it completes in time $C_{i}^{*}$, earlier than scheduled. Note that in this case, all the scheduled work for task $\varphi_{i}$ is completed before the deadline $T_{1}$. Similarly, the average $V D D$ value for task $\varphi_{j}$ decreases below $V D D_{j}$, resulting in a situation where $\varphi_{j}$ completes in time $C_{j}^{*}$ (longer than its its original WCET). However, we must guarantee that all the work for task $\varphi_{j}$ is completed before its deadline $T_{2}$. In other words, we need to find the time $T_{1}^{*}$ to start the $V D D$ transition, such that the speed-up of task $\varphi_{i}$ is equal to the slow-down of task $\varphi_{j}$. Therefore, we need to find $T_{1}^{*}$ such that:

$$
C_{i}-C_{i}^{*}=C_{j}^{*}-C_{j}=D
$$

In this manner, we can perform computation while $V D D$ is being switched, allowing us to reduce the pessimism in the WCETs of the tasks. The VDD switching is therefore performed on-the-fly, even while tasks $\varphi_{i}$ and $\varphi_{j}$ are being computed.

If $V D D_{i}>V D D_{j}$, then by starting the transition at the same time $T_{1}$ as in the ideal case of Figure 2a), we can ensure that the work of $\varphi_{i}$ is completed before its deadline $T_{1}$. For $\varphi_{2}$, the average $V D D$ value is above $V D D_{j}$, and hence it completes earlier than scheduled, again guaranteeing that its work was completed before its deadline $T_{2}$.

The computation of $T_{1}^{*}$ is performed as follows.

The total delay of computing tasks $\varphi_{i}$ and $\varphi_{j}$ using our on-the-fly VDD switching methodology is given by Equation 2.

$$
\begin{aligned}
N_{\text {ours }} & =\int_{0}^{T_{1}^{*}} F\left[V D D_{i}\right] d t+ \\
& \int_{T_{1}^{*}}^{T_{2}} F\left[V D D_{j}+\left(V D D_{i}-V D D_{j}\right) e^{\frac{-\left(t-T_{1}^{*}\right)}{\tau}}\right] d t
\end{aligned}
$$

Here $F(x)$ is the frequency of operation of the embedded processor when the supply value is $V D D=x$ volts. $N_{\text {ours }}$ is the total number of computational cycles that are performed in the interval $\left[0, T_{2}\right]$ by our method. Also, $\tau$ is the $\mathrm{RC}$ time constant of the on-chip $V D D$ network.

Similarly, the total number of computational cycles $N_{\text {ideal }}$ (for the method of Figure 2a) which assumes that the $V D D$ change is a step function) is given by Equation 3 .

$$
N_{\text {ideal }}=\int_{0}^{T_{1}} F\left[V D D_{i}\right] d t+\int_{T_{1}}^{T_{2}} F\left[V D D_{j}\right] d t
$$


cording to this model, the delay of a MOS circuit as a function of VDD is given by Equation 4. Note that we assume that if the supply voltage is $V_{D D}$, then the frequency is inversely proportional to $D\left(V_{D D}\right)$. In other words, frequency is not an independent variable, and is coupled to the value of $V_{D D}$ utilized.

$$
D\left(V_{D D}\right)=K \cdot \frac{V_{D D}}{\left(V_{D D}-V_{T}\right)^{\alpha}}
$$

The first task we performed was to find the value of $\alpha$. We determined through experimentation that $\alpha=2$. We used a simple 9-stage ring oscillator, along with two other circuits (apex7 and alu4) from the MCNC91 benchmark suite. We mapped these circuits using SIS [20] to a library of 21 gates using a predictive $0.1 \mu \mathrm{m}$ process technology [21]. The results are shown in Figure 3, which plots the value of $\frac{V D D}{\left(V D D-V_{T}\right)^{2}}$ against delay. This shows an accurate fit for $\alpha=2$.

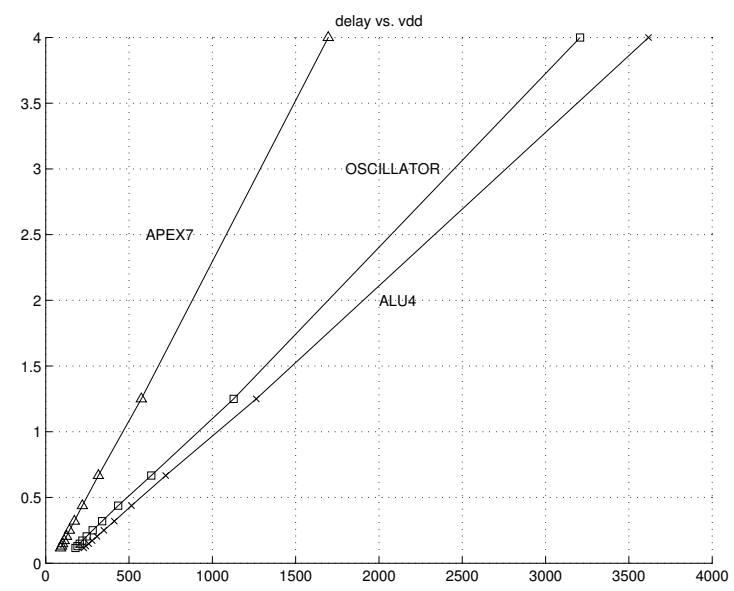

Figure 3: Finding the value of $\alpha$ in Equation 4

Therefore, the processor delay (and consequently its frequency) is:

$$
\begin{gathered}
D\left(V_{D D}\right)=K \cdot \frac{V_{D D}}{\left(V_{D D}-V_{T}\right)^{2}} \\
F\left(V_{D D}\right)=K^{\prime} \cdot \frac{\left(V_{D D}-V_{T}\right)^{2}}{V_{D D}}
\end{gathered}
$$

By simply plugging Equation 6 into Equations 3 and 2, we have the following two equations that compute the number of instruction executed in a time period, (in this case, $\left[0, T_{2}\right]$ ) with and without taking into account of the $V_{D D}$ switching delay. We assume in the sequel that the VDD change is from a value $V_{a}$ to $V_{b}$ (where $V_{a}<V_{b}$ ).

If $V_{D D}$ switching is a step function (the ideal case of Figure 2a)), we have:

$$
\begin{aligned}
N_{\text {ideal }} & =\int_{0}^{T_{1}} F\left(V_{a}\right) d t+\int_{T_{1}}^{T_{2}} F\left(V_{b}\right) d t \\
& =K^{\prime} \cdot\left\{\int_{0}^{T_{1}} \frac{\left(V_{a}-V_{T}\right)^{2}}{V_{a}} d t+\int_{T_{1}}^{T_{2}} \frac{\left(V_{b}-V_{T}\right)^{2}}{V_{b}} d t\right\} \\
& =K^{\prime} \cdot\left\{\frac{\left(V_{a}-V_{T}\right)^{2}}{V_{a}} \cdot T_{1}+\frac{\left(V_{b}-V_{T}\right)^{2}}{V_{b}} \cdot\left(T_{2}-T_{1}\right)\right\} \\
& =K^{\prime} \cdot\left\{\frac{\left(V_{b}-V_{T}\right)^{2}}{V_{b}} \cdot T_{2}+\left[\frac{\left(V_{a}-V_{T}\right)^{2}}{V_{a}}-\frac{\left(V_{b}-V_{T}\right)^{2}}{V_{b}}\right] \cdot T_{1}\right\}
\end{aligned}
$$

Now let us account for the VDD switching delay (the case of Figure 2b)). From $T_{1}^{*}$ to $T_{2}, V_{D D}$ is an exponential function of time:

$$
\begin{aligned}
V_{D D}(t) & =V_{b}+\left(V_{a}-V_{b}\right) \cdot e^{-\frac{t}{\tau}} \\
& =A+B \cdot e^{-\frac{t}{\tau}}
\end{aligned}
$$

where $\mathrm{A}=V_{b}$ and $\mathrm{B}=V_{a}-V_{b}$. Substituting Equation 8 in Equation 2, and assuming that $T_{2} \gg \tau$, we get:

$$
\begin{aligned}
N_{\text {ours }}= & \int_{0}^{T_{2}} F(V(t)) d t \\
= & K^{\prime} \cdot\left(\int_{0}^{T_{1}^{*}} \frac{\left(V_{a}-V_{T}\right)^{2}}{V_{a}} d t+\right. \\
& \left.\int_{T_{1}^{*}}^{T_{2}} \frac{\left(A+B \cdot e^{-\frac{t}{\tau}}-V_{T}\right)^{2}}{A+B \cdot e^{-\frac{t}{\tau}}} d t\right)
\end{aligned}
$$

Without loss of generality, we let $T_{1}^{*}=0$. In that case, $T_{1}$ becomes the look-ahead time, which we need to determine:

Let $t^{\prime}=t / \tau$ and $T_{2}^{\prime}=T_{2} / \tau$, and using the assumption that $T_{2} \gg \tau$, we get:

$$
\begin{aligned}
N_{\text {ours }}= & \tau K^{\prime} \int_{0}^{T_{2}^{\prime}}\left(A+B e^{-t^{\prime}}-2 V_{T}+\frac{V_{T}^{2}}{A+B e^{-t^{\prime}}}\right) d t^{\prime} \\
= & \tau K^{\prime}\left\{A T_{2}^{\prime}-2 V_{T} T_{2}^{\prime}+B\left(1-e^{-T_{2}^{\prime}}\right)\right. \\
& \left.+\frac{V_{T}^{2}}{A}\left[T_{2}^{\prime}+\ln (A)-\ln (A+B)\right]\right\} \\
= & \tau K^{\prime}\left\{\frac{\left(A-V_{T}\right)^{2}}{A} T_{2}^{\prime}\right. \\
& \left.+\left[B+\frac{V_{T}^{2}}{A}(\ln (A)-\ln (A+B))\right]\right\} \\
= & K^{\prime} \frac{\left(A-V_{T}\right)^{2}}{A} T_{2} \\
& +\tau K^{\prime}\left[B+\frac{V_{T}^{2}}{A} \cdot(\ln (A)-\ln (A+B))\right] \\
= & K^{\prime} \frac{\left(V_{b}-V_{T}\right)^{2}}{V_{b}} T_{2} \\
& +\tau K^{\prime}\left[V_{a}-V_{b}+\frac{V_{T}^{2} \cdot \ln \left(V_{b}\right)-\ln \left(V_{a}\right)}{V_{b}}\right]
\end{aligned}
$$

To find the time $T_{1}$ (look-ahead time) that makes $N_{\text {ours }}=N_{\text {ideal }}$, we combine Equation 9 and Equation 7 to get:

$$
\begin{aligned}
& K^{\prime} \cdot\left\{\frac{\left(V_{b}-V_{T}\right)^{2}}{V_{b}} \cdot T_{2}+\left[\frac{\left(V_{a}-V_{T}\right)^{2}}{V_{a}}-\frac{\left(V_{b}-V_{T}\right)^{2}}{V_{b}}\right] \cdot T_{1}\right\} \\
& =K^{\prime} \frac{\left(V_{b}-V_{T}\right)^{2}}{V_{b}} \cdot T_{2}+\tau K^{\prime}\left(V_{a}-V_{b}+\frac{V_{T}^{2}\left(\ln \left(V_{b}\right)-\ln \left(V_{a}\right)\right)}{V_{b}}\right)
\end{aligned}
$$

The first terms on both sides cancel each other, making the resulting equation independent of $T_{2}$, yielding:

$$
T_{1}=\frac{V_{a}-V_{b}+\frac{V_{T}^{2}}{V_{b}}\left(\ln \left(V_{b}\right)-\ln \left(V_{a}\right)\right)}{\frac{\left(V_{a}-V_{T}\right)^{2}}{V_{a}}-\frac{\left(V_{b}-V_{T}\right)^{2}}{V_{b}}} \cdot \tau
$$

As we can see, $T_{1}$ is independent of $T_{2}$, which is intuitively reasonable. The above expression for $T_{1}$ is true when we assume $T_{2} \gg \tau$. Note that if the $T_{2} \gg \tau$ assumption does not hold, then a closed form expression for $T_{1}$ can still be computed. In this case, $T_{1}$ has a dependence on $T_{2}$ as well.

$T_{1}$ is dependent on $V_{T}$ and the $V_{D D}$ of both tasks $\varphi_{i}$ and $\varphi_{j}$. Also in reality, $V_{a}$ and $V_{b}$ are greater than $V_{T}$ for circuits that operate above threshold voltage. Today's processors all utilize super-threshold conduction to perform their computations.

From Equation 11, the lower bound of $T_{1}=\tau$ is achieved when $V_{T}=0$. This bound is not very meaningful in practice, since $V_{T}=0$ would result in extremely high leakage currents in the design.

When both $V_{a}$ and $V_{b}$ are much greater than $V_{T}, T_{1}$ is very close to $\tau$.

Table 1 reports the calculated look-ahead delay $T_{1}$ (in multiples of $\tau$ ) for several values of $V_{a}$ and $V_{b}$. For this table, we used $V_{T}=0.3 \mathrm{~V}$.

Assuming the circuit is operating at or above threshold voltage $V_{T}$, we can determine from Equation 11 that the upper bound of $T_{1}$ is $1.494 \cdot \tau$. This upper bound is achieved when $V_{a}=V_{T}$. If $V_{a}$ is no lower than $0.4 \mathrm{~V}$ and $V_{T}=0.3 \mathrm{~V}$ (reasonable values for modern embedded processors) the upper bound drops to $1.12 \cdot \tau$. In practice, we would compute the look ahead time $5117 T_{1}$ on-the-fly, or simply obtain it by performing a table look-up. This is 


\begin{tabular}{|c||c|c|c|c|c|c|c|c|}
\hline$V_{a} \backslash V_{b}$ & 0.35 & 0.4 & 0.6 & 0.8 & 1.0 & 1.2 & 1.4 & 1.6 \\
\hline \hline 0.35 & - & 1.175 & 1.184 & 1.169 & 1.150 & 1.134 & 1.121 & 1.110 \\
\hline 0.4 & 0.877 & - & 1.113 & 1.120 & 1.113 & 1.104 & 1.096 & 1.088 \\
\hline 0.6 & 0.780 & 0.870 & - & 1.032 & 1.041 & 1.044 & 1.044 & 1.043 \\
\hline 0.8 & 0.778 & 0.849 & 0.965 & - & 1.014 & 1.020 & 1.022 & 1.023 \\
\hline 1.0 & 0.787 & 0.847 & 0.951 & 0.985 & - & 1.007 & 1.011 & 1.013 \\
\hline 1.2 & 0.798 & 0.851 & 0.945 & 0.978 & 0.992 & - & 1.004 & 1.007 \\
\hline 1.4 & 0.809 & 0.856 & 0.942 & 0.973 & 0.988 & 0.996 & - & 1.003 \\
\hline 1.6 & 0.819 & 0.861 & 0.941 & 0.971 & 0.985 & 0.993 & 0.997 & - \\
\hline
\end{tabular}

Table 1: Look-ahead time $T_{1}$ as a function of $V_{a}$ and $V_{b}$ (multiples of $\tau$ )

feasible if $V_{D D}$ has a limited number of discrete values. In practice, however, it may be more attractive to use a fixed upper bound value for simplicity of implementation.

It should be noted that our technique may only be applied under two conditions. First, adjacent tasks $\varphi_{i}$ and $\varphi_{j}$ should be such that the task $\varphi_{j}$ is queued before time $T_{1}^{*}$. Secondly, the quantity $C_{j}^{*}$ should be such as to allow the $V D D_{i}$ to switch completely to $V D D_{j}$. Of course since the switching characteristic is an exponential waveform, it is sufficient in practice for the transition to be greater than $99 \%$ complete (which requires that $C_{j}^{*}>4.6 \times \tau$ ). This is easy to check statically once a schedule is available.

If $V_{a}>V_{b}$, then the situation is simpler: If $\varphi_{i}$ has a WCET less than the original switching time, then Table 1 is used to determine the look-ahead time (assuming the deadline of both tasks is $T_{2}$.) If the task $\varphi_{i}$ has a WCET which is equal to $T_{1}$, then the switching of VDD must be performed at the originally scheduled time (so that the work of $\varphi_{i}$ can be guaranteed to complete). Since the average value of VDD is higher during the computation of $\varphi_{j}$, it completes earlier than scheduled. Once again, we ensure that the required work for $\tau_{i}$ and $\tau_{j}$ is completed before their respective deadlines. We can use our method (analogous equations can be derived for the $V_{a}>V_{b}$ case) to determine how much earlier $\varphi_{j}$ will complete. The remaining time before the deadline $T_{2}$ can be used to put the processor into a sleep state, further reducing power. Alternately, the supply voltage $V_{b}$ can be recomputed, allowing for further power reduction.

Utilizing our method: Our approach can be utilized for static as well as dynamic schedules. All that is required to be known to find the lookahead time is the value of $V_{a}, V_{b}, \tau$ and $T_{2}$. The discussions above indicate the constraints that must be satisfied when applying our methodology. The computation of look-ahead time is performed at least 1.494. $\tau$ before a deadline. A simple table lookup (which is inexpensive in terms of computation time and power) yields the value of look-ahead time. In case $T_{2} \gg \tau$, then the look-ahead time is independent of $T_{2}$.

\section{Experimental Results}

To verify the correctness of our analysis, we ran some SPICE [22] simulations using the computed value of the look ahead time $T_{1}$ (using Equation 11). We switched the VDD signal at this time instant, and computed the total number of cycles $\left(N_{\text {ours }}\right)$ processed by the embedded processor in the interval $\left[0, T_{2}\right]$. We compared this with the number of cycles $N_{\text {ideal }}$ processed in the same interval when VDD switches in an ideal manner. We ran the simulation for $3 \mu \mathrm{s}$, which is about $6 \cdot \tau$. We again used the 9 -inverter ring oscillator circuit as a reference design.

Table 2 lists the simulation results for some typical $V_{D D}$ values. As indicated in this table, the absolute maximum error is less than 15 clock cycles. This translates to a worst-case error of less than $0.2 \%$ when our method is employed. In practice, a small guardband on the value of the look-ahead time would avoid this small error.

\begin{tabular}{|c|c|c|c|c|c|c|}
\hline$V_{a}(\mathrm{~V})$ & $V_{b}(\mathrm{~V})$ & $\tau(\mathrm{ns})$ & $T_{1}(\mathrm{~ns})$ & $N_{i d e a l}$ & $N_{\text {ours }}$ & $\operatorname{Err}(\%)$ \\
\hline 0.4 & 0.8 & 500 & 525.5 & 4324 & 4317 & 0.2 \\
\hline 0.4 & 1.0 & 500 & 556.5 & 6480 & 6495 & 0.1 \\
\hline 0.4 & 1.2 & 500 & 548 & 6994 & 7010 & 0.2 \\
\hline 0.6 & 0.8 & 500 & 516 & 4497 & 4490 & 0.2 \\
\hline 0.6 & 1.0 & 500 & 520.5 & 7010 & 6994 & 0.2 \\
\hline 0.6 & 1.2 & 500 & 522 & 8755 & 8747 & 0.1 \\
\hline 1.0 & 1.4 & 500 & 505.5 & 11324 & 11310 & 0.1 \\
\hline
\end{tabular}

\section{Table 2: Validation of our Approach with SPICE Simulations}

The close match between the simulation results and our computed value indicates that our analysis is accurate.

\section{Conclusions}

In recent times, the ubiquity of low-power real-time embedded systems has opened up several interesting research problems in scheduling algo5118 rithms for such systems in a power-aware context. To achieve this, several research papers utilize dynamic voltage scaling (DVS) of the power supply. However, the delay incurred during the switching of the $V D D$ signal has not been addressed in these works. As a result, the worst case VDD switching delay is implicitly included in the WCET of each task, leading to an increased pessimism in the WCETs of tasks.

In this paper, we have described a technique to perform on-the-fly DVS. In our approach, computation continues while the VDD is being switched. This allows us to have more realistic WCET estimates for tasks, and maximally utilize the available computation time. In our approach, when there are two tasks $\varphi_{i}$ and $\varphi_{j}$ scheduled consecutively with supply values $V_{D D_{i}}$ and $V_{D D j}$, in a manner that $\varphi_{j}$ is already queued, then we switch $V_{D D}$ on-the-fly during the execution of $\varphi_{i}$. We have derived the expression for the time instant that the $V_{D D}$ switching must begin during the execution of $\varphi_{i}$. Our model was validated with SPICE simulations, and has a maximum error of $0.2 \%$ over a variety of switching conditions.

In the future, we plan to extend the application of our ideas to the determination of when to invoke sleep and idle modes for embedded processors.

\section{References}

[1] C. Liu and J. Layland, "Scheduling algorithms for multiprogramming in a hard-real-time environment," Journal of the Association of Computing Machinery, vol. 20, pp. 46-61, Jan 1973.

[2] Y. Shin and K. Choi, "Power conscious fixed priority scheduling for hard real-time systems," in Proceedings, Design Automation Conference, pp. 134-139, 1999.

[3] I. H. D. Kirovski, Q. Gang, M. Potkonjak, and M. Srivastava, "Power optimization of variable-voltage core-based systems," in IEEE Transactions on Computer-Aided Design of Integrated Circuits and Systems, vol. 18, pp. 1702-1714, 1999.

[4] G. Quan and X. Hu, "Energy efficient fixed-priority scheduling for real-time systems on variable voltage processors," in Proceedings, Design Automation Conference, pp. 828-833, June 2001.

[5] M. Schmitz, B. Al-Hashimi, and P. Eles, "Energy-efficient mapping and scheduling for dvs enabled distributed embedded systems," in Proceedings, Design, Automation and Test in Europe Conference and Exhibition, pp. 514-521, March 2002.

[6] W. Kim, J. Kim, and S. Min, "Dynamic voltage scaling algorithm for dynamic-priority hard real-time systems using slack time analysis," in Proceedings, Design, Automation and Test in Europe Conference and Exhibition, pp. 788-794, March 2002.

[7] Y.-H. Lee and C. Krishna, "Voltage-clock-scaling adaptive scheduling techniques for low power in hard real-time systems," in Proceedings. Sixth IEEE Real-Time Technology and Applications Symposium, pp. 156-165, 2000.

[8] J. Luo and N. Jha, "Static and dynamic variable voltage scheduling algorithms for realtime heterogeneous distributed embedded systems," in Proceedings, Asia and South Pacific Design Automation Conference (ASP-DAC), pp. 719-726, Jan 2002.

[9] J. Luo and N. Jha, "Power-profile driven variable voltage scaling for heterogeneous distributed real-time embedded systems," in Proceedings, International Conference on VLSI Design, pp. 369-375, Jan 2003.

[10] V. Culver and S. Khatri, "Dynamic voltage scaling algorithm for energy reduction in hard real-time systems," in Proceedings, Asia South Pacific Design Automation Conference (ASP$D A C$ ), (Shanghai, China), January 2005.

[11] J. Luo and N. Jha, "Power-conscious joint scheduling of periodic task graphs and aperiodic tasks in distributed real-time embedded systems," in Proceedings, IEEE/ACM International Conference on Computer Aided Design, pp. 357-364, Nov 2000.

[12] V. Gutnik and A. Chandrakasan, "An efficient controller for variable supply-voltage low power processing," in Proceedings, Symposium on VLSI Circuits, pp. 158-159, 1996.

[13] W. Namgoong, M. Yu, and T. Meng, "A high-efficiency variable-voltage CMOS dynamic DC-DC switching regulator," in IEEE International Solid State Circuits Conference, pp. 380-381, 1997.

[14] W. Bowhill, R. Allmon, S. Bell, E. Cooper, D. Donchin, J. Edmondson, T. Fischer, P. Gronowski, A. Jain, P. Kroesen, B. Loughlin, R. Preston, P. Rubinfeld, M. Smith, S. Thierauf, and G. Wolrich, "A $300 \mathrm{mhz} 64$ b quad-issue cmos risc microprocessor," in Digest of Technical Papers, IEEE International Solid-State Circuits Conference, pp. 182-183, Feb 1995

[15] N. Jha, "Low power system scheduling and synthesis," in Proceedings, IEEE/ACM International Conference on Computer Aided Design, pp. 259-263, Nov 2001.

[16] J. Luo and N. Jha, "Battery-aware static scheduling for distributed real-time embedded systems," in Proceedings, Design Automation Conference, pp. 444-449, June 2001.

[17] "Intel XScale Technology." http://www.intel.com/design/intelxscale/.

[18] "Transmeta crusoe overview." http://www.transmeta.com/crusoe/.

[19] T. Sakurai and A. Newton, "Alpha-power law MOSFET model and its application to CMOS inverter delay and other forumals," in IEEE Transactions on Solid State Circuits, vol. 25, pp. 584-594, 1990.

[20] E. M. Sentovich, K. J. Singh, L. Lavagno, C. Moon, R. Murgai, A. Saldanha, H. Savoj, P. R. Stephan, R. K. Brayton, and A. L. Sangiovanni-Vincentelli, "SIS: A System for Sequential Circuit Synthesis," Tech. Rep. UCB/ERL M92/41, Electronics Research Lab, Univ. of California, Berkeley, CA 94720, May 1992

[21] "BSIM3 Homepage." http://www-device.eecs . berkeley. edu/ bsim3/intro.html.

[22] L. Nagel, "Spice: A computer program to simulate computer circuits," in University of California, Berkeley UCB/ERL Memo M520, May 1995. 